\title{
Game Analysis of the Knowledge Sharing Mechanism for the Supply Chain Collaborative Innovation
}

\author{
Liang Liu, Guo Chen*, Xiaoge Niu \\ College of Management and Economics, Tianjin University (China) \\ liuliang@,tju.edu.cn,*Corresponding author amigofishchen@163.com,646401694@,qq.com
}

Received: October 2014

Accepted: March 2015

\section{Abstract:}

Purpose: In information economy era, innovation is the key to improve the competitiveness of enterprises. The traditional way of enterprise innovation is outdated and supply chain collaborative innovation has becoming popular. This paper aims to analyze the mechanism of knowledge sharing between enterprises in supply chain collaborative innovation.

Design/methodology/approach: This paper analyzes the supply chain members' willingness to share knowledge by using the game theory. The result of knowledge sharing between two companies is analyzed by using the evolutionary game.

Findings: We broke the knowledge sharing process in supply chain collaborative innovation into knowledge mining and knowledge transferring. We got the best knowledge sharing strategy of each supply chain member. We gave the influencing factors of knowledge sharing between members for the knowledge sharing mechanisms in supply chain collaborative innovation.

Research limitations/implications: We didn't study the willingness of more than two supply chain members to share knowledge and the result of knowledge sharing between them. And this situation is more realistic.

Practical implications: Our findings can help to improve the effect of knowledge sharing in supply chain collaborative innovation. 
Originality/value: The paper introduces the game theory to knowledge sharing between members in supply chain collaborative innovation, deepens the understanding of knowledge sharing in supply chain collaborative innovation, and gives some interesting findings.

Keywords: game theory, knowledge sharing, collaborative innovation, supply chain

\section{Introduction}

From the late 20th century to the early 21st century, the rapid development of computer technology, especially the rapid application of the internet, has made the transfer and the sharing of information more quickly and easily. A supply chain constitutes of several enterprises that cooperate with each other. The development of the information technology has accelerated the transmission of information between all enterprises in the supply chain and facilitates knowledge sharing between them. Moreover, in information economy era, innovation is the key to improve the competitiveness of the enterprise. However, the enterprise innovates all by itself cannot bring the innovation of the entire supply chain, which brings supply chain collaborative innovation. Supply chain collaborative innovation means in order to improve the market competitiveness and interests of the supply chain, all supply chain members, including suppliers, manufacturers, vendors, etc., innovate together and collaborate with each other in production, logistics, and marketing.

Obviously, supply chain collaborative innovation occurred among many companies and it has many special problems compared with the enterprise innovates alone. The most important problems are listed below. The first problem is the distribution of benefits. The most important purpose of supply chain collaborative innovation is to promote the interests of supply chain members. However, each enterprise wants to maximize its own profit. Then, how to distribute benefits among all supply chain members is the most important problem. The second problem is the integration of different corporate cultures. Each enterprise has its own unique corporate culture, and supply chain collaborative innovation requires companies to work together, then it is important for supply chain members to integrate their cultures. The third problem is knowledge sharing between supply chain members. Knowledge sharing within an enterprise has always been a big problem. And knowledge sharing between supply chain members is a more difficult problem. In order to solve these problems, this paper analysis the knowledge sharing mechanism of enterprises in the supply chain collaborative innovation. 


\section{Related Research}

The traditional way of enterprise innovation is enterprise innovates all by itself. However, this way cannot accelerate the operational efficiency of the entire supply chain and may also increase the cost of the upstream and downstream enterprises. Therefore, scholars began to study supply chain collaborative innovation. Bello et al. believed all supply chain members must participate in collaborative innovation and build management model of collaborative innovation (Bello, Lohtia \& Sangtani, 2004). Choi et al. analyzed all aspects of the supply chain and found that the efficiency and cost of a supply chain have a certain relationship to collaborative innovation (Choi \& Krause, 2006).

In order to achieve collaborative innovation, it is necessary for supply chain members to build a knowledge alliance and share knowledge with each other. Scholars defined knowledge sharing from the following angles.

\subsection{The Flow of Knowledge}

Holthouse thought knowledge is a flow and knowledge sharing is the exchange process between knowledge owners and knowledge recipients (Holthouse, 2006). Hendriks proposed the knowledge sharing process is the communication process among supply chain members. And each member should have its own knowledge that can be shared with others (Hendriks, 1999). Lee thought that knowledge sharing is knowledge transferred from an individual or organization to another individual or organization (Lee, 2001). Bartol and Srivastava believed knowledge sharing is employees delivering and transferring relevant information in the enterprise (Batrol \& Srivastave, 2002). Connelly and Kelloway found in addition to exchange of information, knowledge sharing also can help other companies in many ways (Connelly \& Kelloway, 2003).

\subsection{Information Exchange}

Tan proposed that sharing is exchanging (Tan, 1994). Davenport and Prusak compared the process of knowledge sharing to commodities exchanging, which contains buyers and sellers that can exchange knowledge in the knowledge market (Davenport \& Prusak, 1999).

\subsection{Organizational Learning}

Senge believed that knowledge sharing is not just the transmission of knowledge, but also includes the process of absorption of the received knowledge (Senge, 2006). Dixon (2000) 
thought knowledge sharing is not only means knowledge is accepted to both sides but also means knowledge is accepted to all organizations (Dixon, 2000).

Currently, scholars have no uniform definition of the knowledge sharing mechanism. O'Dell and Grayson believed that knowledge sharing is composed of four parts; they are information system, organizational structure, corporate culture, and evaluation system (O'Dell \& Grayson, 1998). Ruggles found corporate culture is the biggest factors hindering knowledge sharing (Ruggles, 1998).

\section{Knowledge Sharing Process in Supply Chain Collaborative Innovation}

According to Hansen's knowledge-sharing process stage, we break knowledge sharing process in supply chain collaborative innovation into knowledge mining and knowledge transferring.

\subsection{Knowledge Mining}

In supply chain collaborative innovation, knowledge mining refers to supply chain members to explore the knowledge source. On one hand, main sources of knowledge are the market and customers, and knowledge about the market and customers is generally provided by the vendors. Because in the supply chain, vendors is the closest to the customers, and they are easy to understand market situation and customer needs.

On the other hand, the goal of supply chain collaborative innovation is to reduce cost, which is related to each supply chain member. Only under the circumstance of collaborative innovation can all supply chain members minimize cost, otherwise there may be only a single enterprise can reduce its cost. In the case of reduce cost, all supply chain members are sources of knowledge.

Thus, each supply chain members may be the source of knowledge. An enterprise provides knowledge to another enterprise is the knowledge mining process, which is the first stage of knowledge sharing. Because the prerequisite of knowledge sharing is the knowledge source is willing to provide knowledge. Only when it is willing to provide knowledge, the knowledge sharing activities can continue. Therefore, we must understand the distribution of knowledge in supply chain members. 


\subsection{Knowledge Transferring}

Knowledge transferring is the second step of knowledge sharing. Generally, knowledge transferring is divided into three parts: knowledge transmission, knowledge absorption, and knowledge feedback.

If it is a simple knowledge, after knowledge mining, both sides of knowledge sharing only have to transfer knowledge once. But if it is a complex knowledge, then the process of knowledge transfer must be taken more than once. Moreover, because knowledge is easy to be lost and misinterpreted, sharing knowledge repeatedly becomes more important, which also results in knowledge sources have to continuously provide knowledge to knowledge sharing parties.

Meanwhile, in a number of knowledge transfer and absorption process, parties may incur additional knowledge, the transmission of this knowledge back to the sources of knowledge is knowledge feedback. Repeated knowledge transfer, absorption, and feedback form a complete knowledge transfer process. It is specific shown in Figure 1.

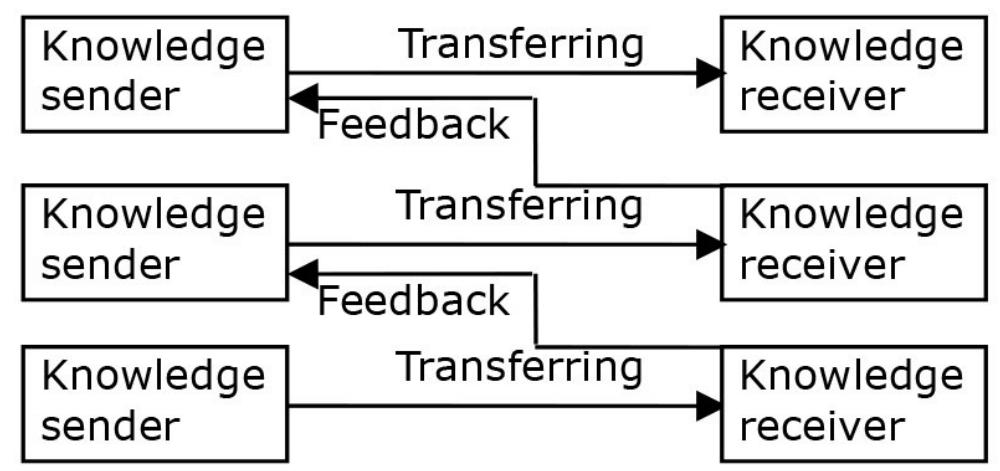

Figure 1. Process of Knowledge Transferring

\section{Prisoner's Dilemma of Knowledge Sharing}

Knowledge sharing in supply chain collaborative innovation environment occurs among all supply chain members. And the goal of each enterprise is to maximize its own interests. Companies game with each other and choose their best knowledge sharing strategies at last.

The willingness of knowledge sharing of each enterprise in the supply chain is not completely transparent, and their knowledge is independent of each other. Especially in the dissemination and sharing of tacit knowledge, supply chain members' game all the time. As the Prisoner's Dilemma, when a participating company does not know whether another participator shares its knowledge or not, it can choose to share or not to share knowledge. Thus, there exist three kinds of results. 
(1) If both two participators choose to share knowledge, both of them can get knowledge provided by the other side. Then they can get the optimal solution $(9,9)$.

(2) If Company A choose to share knowledge, but Company B choose not to share knowledge, Company A will lose a unit payoff while Company B will get an extra unit payoff. Thus, they can get the solution $(-1,10)$. Otherwise, they get the solution $(10,-1)$.

(3) If both Company A and B choose not to share knowledge, both of them cannot get any payoff. Then they only can get the solution $(0,0)$.

The payoff matrix is shown in Table 1.

\begin{tabular}{|c|c|c|}
\hline \multirow{2}{*}{ A } & \multicolumn{2}{|c|}{ B } \\
\cline { 2 - 3 } & To share & Not to share \\
\hline To share & $(9,9)$ & $(10,-1)$ \\
\hline Not to share & $(-1,10)$ & $(0,0)$ \\
\hline
\end{tabular}

Table 1. Payoff Matrix of Company A and B

\subsection{Game analysis}

We assume there are a seller, a manufacturer, and a supplier in a supply chain and they are work together in collaborative product innovation. Since product innovation is mainly done by the manufacturer, then the manufacturer is the leader, the vendor and the supplier are the followers. Specific variables are as follows:

(1) There are 1 leader company and 2 follower companies. Assuming the knowledge production of the leader company is $q_{1}$ and the knowledge production of the follower company is $q_{2}$. And the follower decides its knowledge production based on the knowledge production of the leader. Then, we can get $S:\left(q_{1} \rightarrow q_{2}\right)$.

(2) In the Stackelberg model, the amount of knowledge provided by the company is the cost and the cost of a company is related to its products.

(3) Assuming the inverse demand function is:

$$
(a+b, a+b)
$$

The cost is:

$$
C_{i}=c \cdot q_{i}
$$

$c$ is the fixed cost of per unit production. 
The profit is:

$$
\pi_{i}\left(q_{1}, q_{2}\right)=q_{i}\left(P-C_{i}\right) i=1,2
$$

(4) The knowledge production of the leader is $q_{1}$, and then the best strategy of the follower is:

$$
\operatorname{Max} \pi_{2}\left(q_{1}, q_{2}\right)=q_{2} \cdot\left(a-q_{1}-q_{2}-c\right)
$$

As the follower determines its knowledge production based on the decision of the leader, then when marginal revenue equals marginal cost the follower maximizes its profit. The knowledge production of the follower is:

$$
q_{2}=\frac{1}{2} \cdot\left(a-q_{1}-c\right)=S_{2}\left(q_{1}\right)
$$

(5) The leader knows the follower decides $q_{2}$ based on $q_{1}$, so we can get the profit of the leader based on $q_{2}$ :

$$
\operatorname{Max} \pi_{1}\left(q_{1}, S_{2}\left(q_{1}\right)\right)=q_{1}\left(a-q_{1}-S_{2}\left(q_{1}\right)-c\right)
$$

By first order optimization, we can get:

$$
\begin{gathered}
q_{1}^{*}=\frac{1}{2}(a-c) \\
q_{2}^{*}=S_{2}\left(q_{1}^{*}\right)=\frac{1}{4}(a-c)
\end{gathered}
$$

(6) At last we can get the outcome of the Nash equilibrium.

Balanced Results:

$$
\left(\frac{1}{2}(a-c), \frac{1}{4}(a-c)\right)
$$

Equilibrium:

$$
\left(q_{1}^{*}, S_{2}\left(q_{1}^{*}\right)\right)
$$

By using the Stackelberg model, we find the best strategy of both the leader and the follower is to share knowledge. 


\section{Evolutionary Game of Knowledge Sharing}

In knowledge sharing among companies, each company has two choices: to share knowledge with another company or not. This paper will study the knowledge sharing mechanism between two companies. To study how many equilibriums these two companies can reach, and the probability of each equilibriums, and how to promote knowledge sharing between these two companies is of great significance.

The basic idea of evolutionary game is that the participator with bounded rationality is impossible to find the best strategy trough a game. The participator can find the best strategy only by imitating and improving the strategies of itself and others in the past. Through a long period of imitation and improvement, each participator will tend to find a stable strategy, which is named Evolutionary Stable strategy (ESS). Because the decision maker of each company has bounded rationality in the fact, this paper studies knowledge sharing among companies by using evolutionary game.

When a company does not know whether another participator shares its knowledge or not, it can choose to share or not to share knowledge. Thus, there exist three kinds of results.

(1) If both Company A and B choose not to share knowledge, they only can get the solution $(a, a)$.

(2) If Company A choose to share knowledge, but Company B choose not to share knowledge, Company A will lose $d$ units payoff while Company B will get $c$ units extra payoff. Thus, they can get the solution $(a-d, a+c)$. Otherwise, they get the solution $(a+c, a-d)$.

(3) If both two companies choose to share knowledge, both of them can get knowledge provided by each other. Then they can get the optimal solution $(a+b, a+b)$.

The payoff matrix is shown in Table 2. And we assume there exist $a<d$ and $b<c$.

\begin{tabular}{|l|c|c|}
\hline \multirow{2}{*}{ A } & \multicolumn{2}{|c|}{ B } \\
\cline { 2 - 3 } & To share & Not to share \\
\hline To share & $a+b, a+b$ & $a-d, a+c$ \\
\hline Not to share & $a+c, a-d$ & $a, a$ \\
\hline
\end{tabular}

Table 2. Payoff Matrix of Company A and B 
The expected revenue of $A$ when it choose to share knowledge, the expected revenue of $A$ when it choose not to share knowledge, and the average expected revenue of $A$ are defined in Eq.11, 12, 13:

$$
\begin{gathered}
U_{11}=y \cdot(a+b)+(1-y) \cdot(a-d) \\
U_{12}=y \cdot(a+c)+(1-y) \cdot a \\
\bar{U}_{1}=x \cdot U_{11}+(1-x) \cdot U_{12}
\end{gathered}
$$

The replicator dynamics equation of $A$ is:

$$
F(x)=\frac{\mathrm{d} x}{\mathrm{~d} t}=x \cdot\left(U_{11}-\bar{U}_{1}\right)=x(1-x)[(2 a-d)+(b+c+d) y]
$$

When $F(x)=0$, we can get two stable states: $x=0$ and $x=1$. We assume $y_{D}=\frac{d-2 a}{b+c+d}$, when $0<\frac{d-2 a}{b+c+d}<1$, there exist three cases:

(14a) when $y<y_{D},\left.\frac{\mathrm{d} F(x)}{\mathrm{d} x}\right|_{x=0}<0$, thus $x=0$ is ESS;

(14b) when $y>y_{D},\left.\frac{\mathrm{d} F(x)}{\mathrm{d} x}\right|_{x=1}<0$, thus $x=1$ is ESS;

(14C) when $y=y_{D}, F(x) \equiv 0$, thus all $x$ are ESS.

The expected revenue of $B$ when it choose to share knowledge, the expected revenue of $B$ when it choose not to share knowledge, and the average expected revenue of $B$ are defined in Eq.15, 16, 17:

$$
\begin{gathered}
U_{21}=x \cdot(a+b)+(1-x) \cdot(a-d) \\
U_{2 n}=x \cdot(a+c)+(1-x) \cdot a \\
\bar{U}_{2}=y \cdot U_{2,1}+(1-y) \cdot U_{\eta}
\end{gathered}
$$

The replicator dynamics equation of $B$ is:

$$
G(y)=\frac{\mathrm{d} y}{\mathrm{~d} t}=y \cdot\left(U_{21}-\bar{U}_{2}\right)=y(1-y)[(2 a-d)+(b+c+d) x]
$$


When $G(y)=0$, we can get two stable states: $y=0$ and $y=1$. We assume $x_{D}=\frac{d-2 a}{b+c+d}$, when $0<\frac{d-2 a}{b+c+d}<1$, there exist three cases:

(18a) when $x<x_{D},\left.\frac{\mathrm{d} G(y)}{\mathrm{d} y}\right|_{y=0}<0$, thus $y=0$ is ESS;

(18b) when $x>x_{D},\left.\frac{\mathrm{d} G(y)}{\mathrm{d} y}\right|_{y=1}<0$, thus $y=1$ is ESS;

(18c) when $x=x_{D}, \mathrm{G}(x) \equiv 0$, thus all $y$ are ESS.

The evolutionary game phase diagram of $A$ and $B$ is shown in Figure 2 .

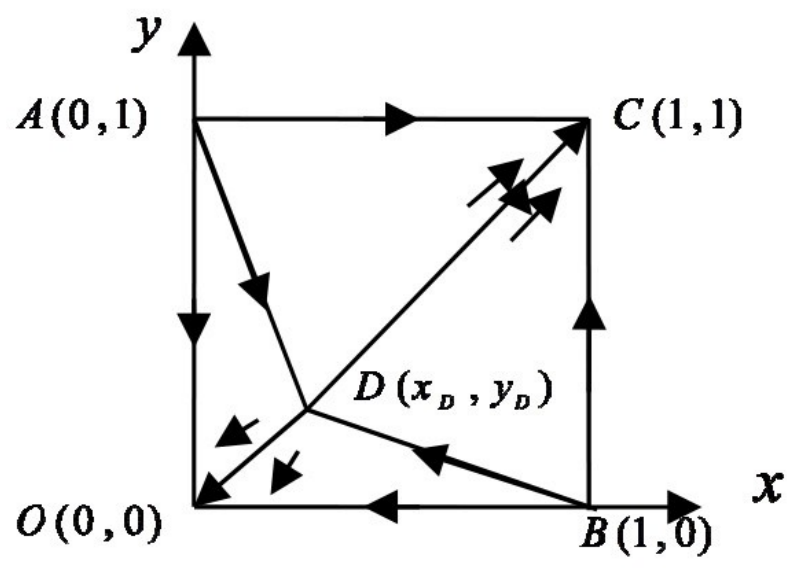

Figure 2. Evolutionary Game Phase Diagram of A and B

The area of $A O B D$ in Figure 2 is:

$$
S_{A O B D}=\frac{1}{2}\left(x_{D}+y_{D}\right)=\frac{d-2 a}{b+c+d}
$$

Obviously, there exist $\frac{\partial S_{A O B D}}{\partial a}<0, \frac{\partial S_{A O B D}}{\partial b}<0, \frac{\partial S_{A O B D}}{\partial c}<0$, and:

$$
\frac{\partial S_{A O B D}}{\partial d}=\frac{1}{b+c+d}\left(1-\frac{d-2 a}{b+c+d}\right)>0
$$

The smaller the area of $A O B D$ is, the greater the probability of $A$ and $B$ finally both choose to share knowledge is. Thus we can promote knowledge sharing between $A$ and $B$ by improving $a, b, c$ and reducing $d$. 
We illustrate this theory by using a simple case. We initially assume that $a=4, b=4, c=6$, and $d=15$. When $a$ is $5,6,7$ while other parameters remain unchanged the evolutionary game phase diagram of system is shown in Figure 3 . When $b$ is $6,8,10$ while other parameters remain unchanged the evolutionary game phase diagram of system is shown in Figure 4 . When $c$ is $8,10,12$ while other parameters remain unchanged the evolutionary game phase diagram of system is shown in Figure 5 . When $d$ is $17,19,21$ while other parameters remain unchanged the evolutionary game phase diagram of system is shown in Figure6.

Figures 3, 4, 5 verifies that the bigger $a, b, c$ is, the smaller the area of AOBD is, that is the greater the probability of $A$ and $B$ finally both choose to share knowledge is. Figure 6 verifies that the smaller $d$ is, the smaller the area of AOBD is, that is the greater the probability of $A$ and $B$ finally both choose to share knowledge is.

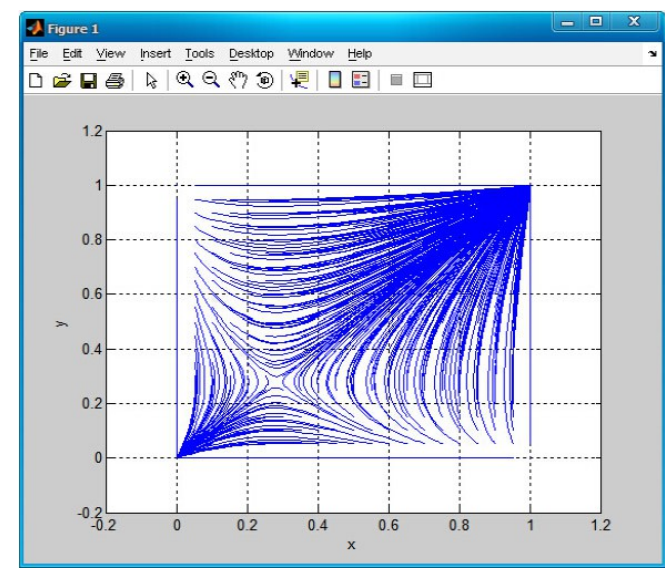

$3-1 a=4, b=4, c=6, d=15$

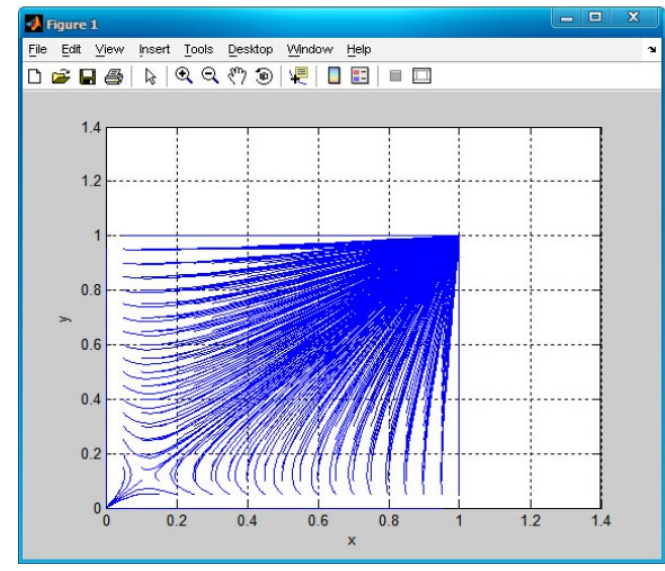

$3-3 a=6, b=4, c=6, d=15$

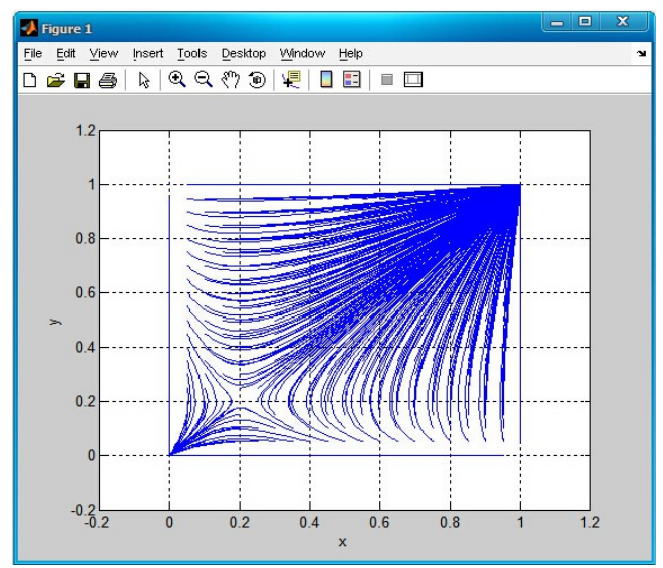

3-2 $a=5, b=4, c=6, d=15$

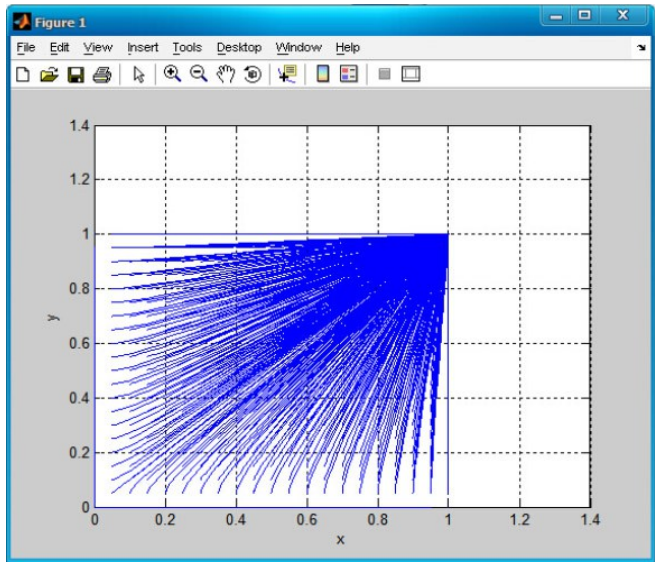

$3-4 a=7, b=4, c=6, d=15$

Figure 3. Evolutionary Game Phase Diagram of System (when $a$ is improved) 


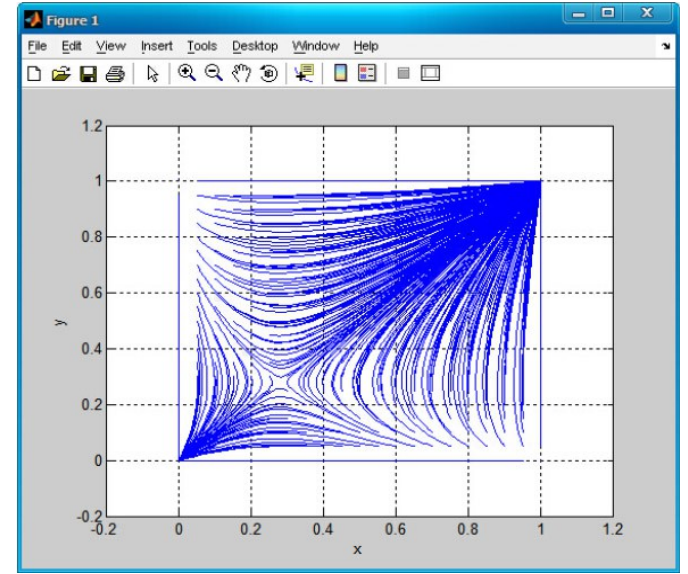

4-1 $a=4, b=4, c=6, d=15$

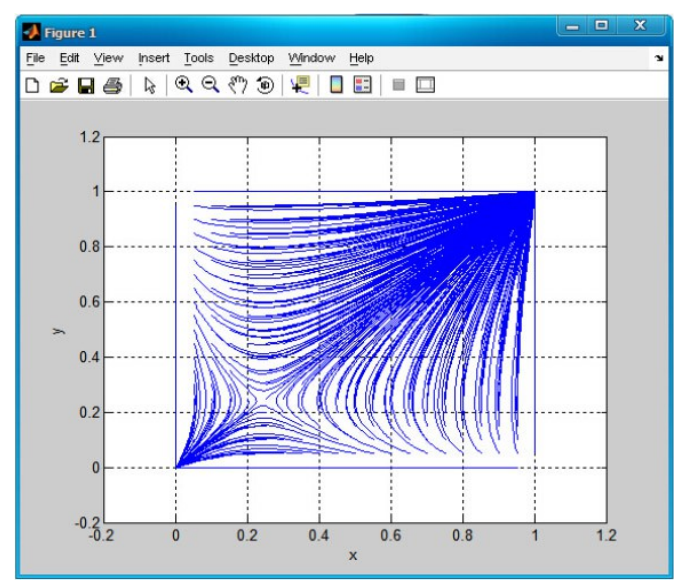

$4-3 a=4, b=8, c=6, d=15$

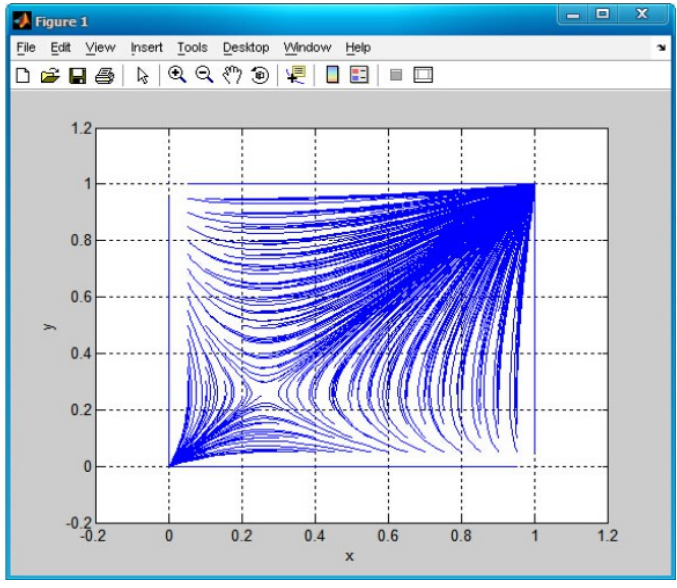

$4-2 a=4, b=6, c=6, d=15$

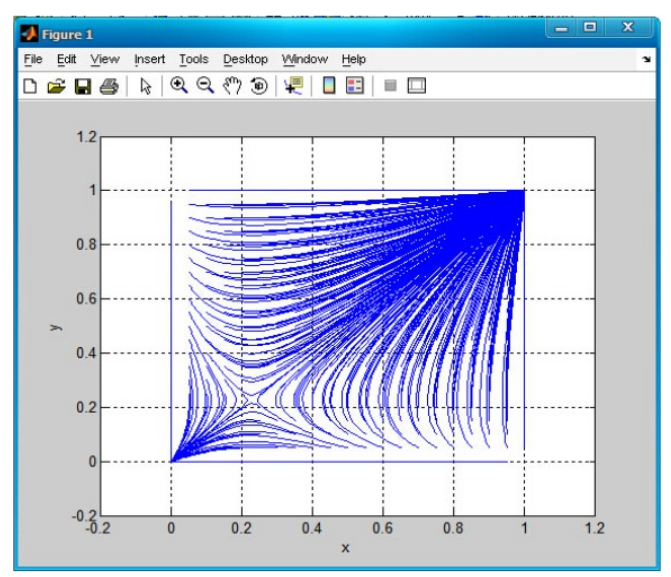

$4-4 a=4, b=10, c=6, d=15$

Figure 4. Evolutionary Game Phase Diagram of System (when $b$ is improved) 


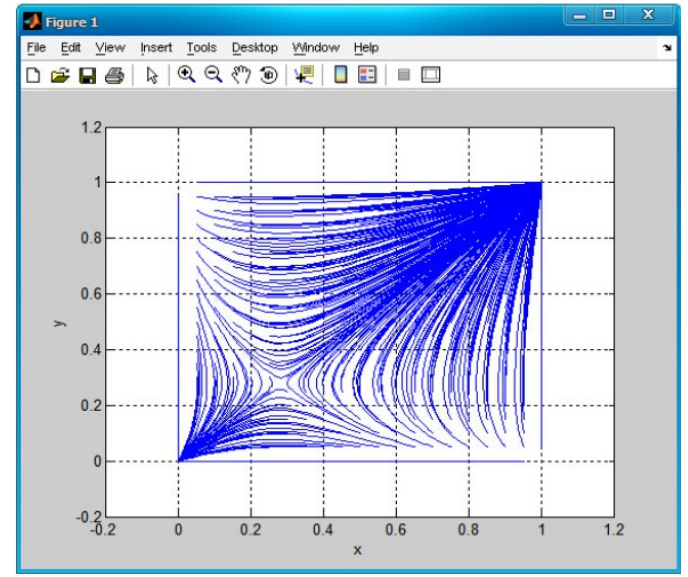

$5-1 a=4, b=4, c=6, d=15$

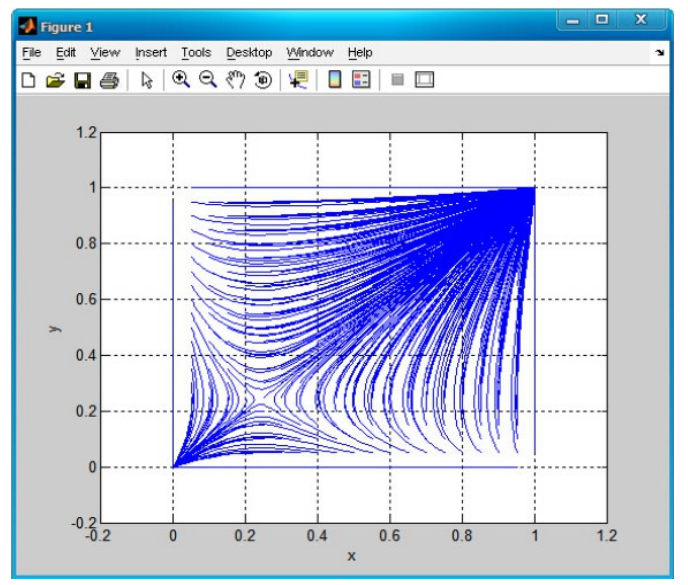

$5-3 a=4, b=4, c=10, d=15$

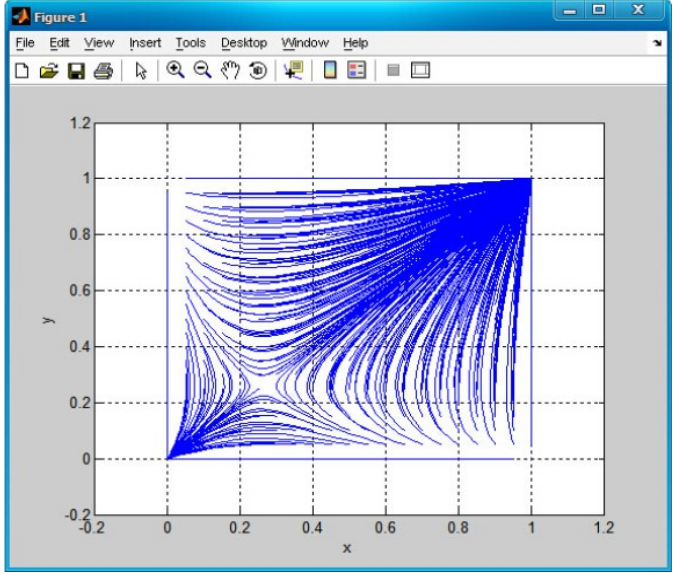

$5-2 a=4, b=4, c=8, d=15$

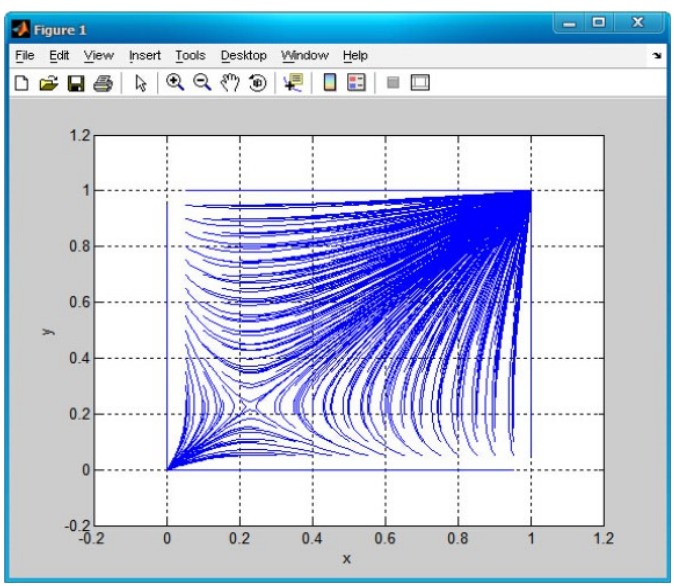

$5-4 a=4, b=4, c=12, d=15$

Figure 5. Evolutionary Game Phase Diagram of System (when $c$ is improved) 


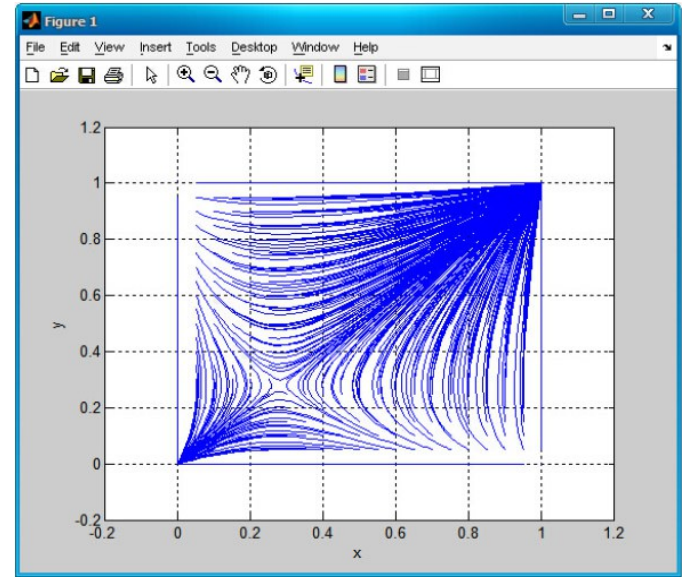

$6-1 a=4, b=4, c=6, d=15$

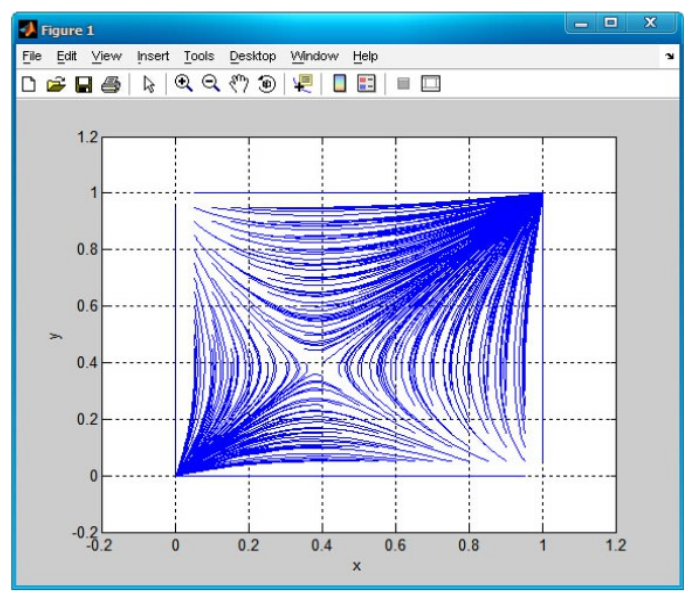

$6-3 a=4, b=4, c=6, d=19$

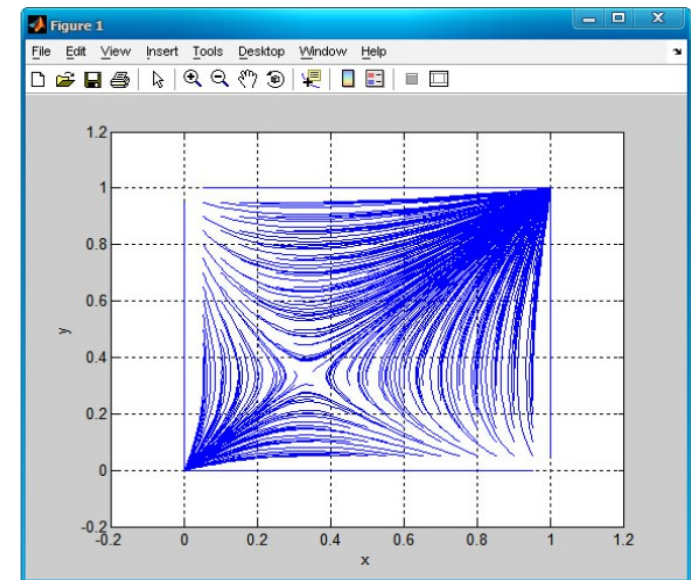

$6-2 a=4, b=4, c=6, d=17$

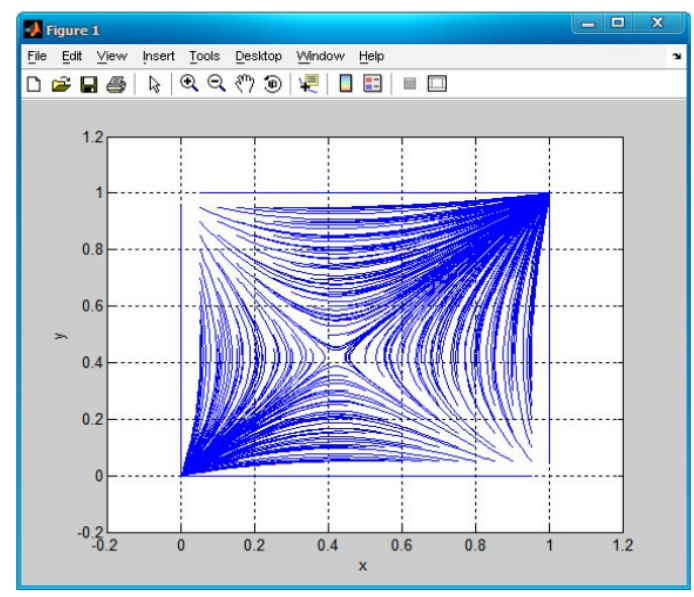

$6-4 a=4, b=4, c=6, d=21$

Figure 6. Evolutionary Game Phase Diagram of System (when $d$ is improved) 


\section{Influencing Factors for Knowledge Sharing}

Many factors may influence knowledge sharing between supply chain members in supply chain collaborative innovation.

\subsection{Trust}

Knowledge sharing occurs between all supply chain members. Li et al. believed trust has a close relationship with the wiliness to share knowledge ( $\mathrm{Li} \& \mathrm{Liu}, 2014$ ). Wang et al. proposed trust is the main reason to promote knowledge sharing. If all members do not trust each other, they cannot share knowledge in the long term (Wang \& Yang, 2012). In most cases, supply chain members care their profits at first and build trust with each other by contracting. But in the supply chain collaborative innovation environment, although there is a collaborative innovation goal, because the result of collaborative innovation may vary according to each different type of collaborative innovation, which leads to companies get different benefits. Therefore, it is hard for supply chain members to trust each other and to provide their entire knowledge, which makes knowledge sharing difficult to continue. Thus, solving the problem of trust between enterprises in the supply chain collaborative innovation environment is a prerequisite and necessary condition of knowledge sharing. Moreover, trust is a kind of emotion, in order to make supply chain members trust each other, employees' thought and emotion are key factors that cannot be ignored.

\subsection{Knowledge Protection}

In order to achieve the goal of collaborative innovation, supply chain members will provide the majority of their knowledge, including their core knowledge. Because the core knowledge is the most important secret of a company, the protection of a company's core knowledge influences the company's wiliness to share knowledge. Wang et al. believed establishing a knowledge protection mechanism can encourage members to share knowledge (Wang \& Zhang, 2008). A company can protect its knowledge by taking the following measures:

(1) When supply chain members sharing their knowledge, not all employees can acquire all the knowledge in the knowledge sharing platform. Employees of different level can get the appropriate kind of knowledge. For example, the general level of corporate employees can only get the general explicit knowledge, which can help them understand the processes of the supply chain. And only the employees whose job is closely related to the core technology can acquire the core knowledge. Moreover, these employees should sign a confidentiality agreement and strictly compliance with it. 
(2) Supply chain members should develop a unified confidential agreement, which specifies how to punish employees who leak knowledge, when they share knowledge with each other. Meanwhile, training employees of each company to develop their sense of confidentiality and make sure they are able to strictly observe the agreement.

\subsection{Cultural Differences}

Although all companies are in the same supply chain, different companies have their own unique corporate culture. Corporate culture is a kind of value or entrepreneurial spirit that is complied by all of the employees in this company. Jin et al. proposed corporate culture influences the company's wiliness to share knowledge (Jin, Yang, Huang \& Wu, 2013). And the cooperation of enterprises needs the integration of corporate cultures, thus, knowledge sharing between supply chain members also requires the integration of corporate cultures.

Meanwhile, corporate culture plays a very important role in knowledge sharing. In 1999, Donoghue, Harris and Weitzman thought different business processes and corporate culture result in different knowledge management methods, which may at last influence the process of knowledge sharing, including knowledge mining and knowledge transferring.

\subsection{Role of Core Enterprise}

In each case of supply chain collaborative innovation, the core enterprise varies depend on different innovation content and innovation subject. For example, in collaborative product innovation, the manufacturer is the core enterprise; it should play as a guide and led the knowledge sharing in the collaborative innovation environment.

\section{Conclusions}

This paper analyses the mechanism of knowledge sharing among enterprises in supply chain collaborative innovation. By reviewing the literatures on supply chain collaborative innovation, knowledge sharing and knowledge sharing mechanism, we break knowledge sharing process in supply chain collaborative innovation into knowledge mining and knowledge transferring. We analyze the number of supply chain members' knowledge production by game theory. And we analyze the result of knowledge sharing between two companies by evolutionary game. Then, we analyze influencing factors of knowledge sharing between supply chain members in supply chain collaborative innovation. The implementation of knowledge sharing mechanisms is 
complex and depends on different facts. Because different supply chains have different traits and demands, it requires researchers to deepen related study.

\section{Acknowledgements}

The authors would like to thank the doctoral scientific Fund Project of the Ministry of Education of China (20120032110035) and the Innovation Method Fund of MOST (2013IM030100).

\section{References}

Batrol, K.M., \& Srivastave, A. (2002). Encouraging knowledge sharing: the role of organizational reward systems. Journal of Leadership \& Organizational Studies, 9(1), 64-76. http://dx.doi.org/10.1177/107179190200900105

Bello, D.C., Lohtia, R., \& Sangtani, V. (2004). An institutional analysis of supply chain innovations in global marketing channels. Industrial Marketing Management, 33, 57-64. http://dx.doi.org/10.1016/j.indmarman.2003.08.011

Choi, T.Y., \& Krause, D.R. (2006). The supply base and its complexity implications for transaction costs, risks, responsiveness, and innovation. Journal of Operations Management, 24(5), 637-652. http://dx.doi.org/10.1016/j.jom.2005.07.002

Connelly, C.E., \& Kelloway, E.K. (2003). Predictors of employee' perceptions of knowledge sharing cultures. Leadership \& Organization Development Journal, 24(5), 294-301. http://dx.doi.org/10.1108/01437730310485815

Davenport, T.H. \& Prusak, L. (1999). Operation knowledge. Nan Chang: Jiang Xi Education Press.

Dixon, N.M. (2000). Common knowledge: how companies thrive on sharing what they know. New York: Harvard University Press.

Hendriks, P. (1999). Why share knowledge? The influence of ICT on the motivation for knowledge sharing. Knowledge and Process Management, 6, 91-100. http://dx.doi.org/10.1002/ (SICI)1099-1441(199906)6:2<91::AID-KPM54>3.0.CO;2-M

Holthouse, D. (2006). Knowledge research issue. California Management Review, 43(3), 277-280.

Jin, H., Yang, Z., Huang, Y.T., \& Wu, J. (2013). Exploring how organizational incentives and organizational culture affect knowledge sharing based on modified SIT. Studies in Science of Science, 31, 1697-1707. 
Lee, J.N. (2001). The impact of knowledge sharing, organizational capability and partnership quality on IS outsourcing success. Information \& Management, 38(5), 323-335. http://dx.doi.org/10.1016/S0378-7206(00)00074-4

Li, W.D., \& Liu, H. (2014). An empirical study on relationship of mutual trust and R\&D professionals' knowledge sharing intentions: the mediating effects of knowledge power loss and reciprocity. Management Review, 26, 128-138.

O'Dell, C., \& Grayson, C.J. (1998). If only we knew what we know: the transfer of internal knowledge and best practice. New York: New York Free Press.

Ruggles, R. (1998). The state of notion: Knowledge management in practice. California Management Review, 40, 80-89. http://dx.doi.org/10.2307/41165944

Senge, P.M. (2006). The fifth discipline: the art and practice of the learning organization. New York: Random House Business.

Tan, M. (1994). Establishing mutual understanding in systems design: an empirical study. Journal of Management Information Systems, 10, 159-182.

Wang, J.R., \& Yang, J. (2012). Relationship of trust, team interaction and knowledge sharing behavior. Science of Science and Management of S. \& T., 33, 31-39.

Wang, L.L., \& Zhang, Y.J. (2008). The interaction between implicit knowledge protection and knowledge sharing in science research team of college. Science and Technology Management Research, 7, 242-243.

Journal of Industrial Engineering and Management, 2015 (www.jiem.org)

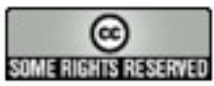

Article's contents are provided on a Attribution-Non Commercial 3.0 Creative commons license. Readers are allowed to copy, distribute and communicate article's contents, provided the author's and Journal of Industrial Engineering and Management's names are included. It must not be used for commercial purposes. To see the complete license contents, please visit http://creativecommons.org/licenses/by-nc/3.0/. 